\title{
Le « jeune » Flaubert et l'apprentissage des genres littéraires
}

\author{
Moulay Youssef SOUSSOU \\ Laboratoire d'études et de recherches sur l'interculturel \\ Université Chouaib Doukkali - Maroc \\ Reçu le 13 mars 2018 | Évalué le 25 avril 2018 | Accepté le 13 juin 2018
}

\begin{abstract}
RÉSUMÉ. L'étude des premiers écrits de Flaubert est un domaine encore inexploré. Une telle étude est tellement salutaire pour saisir la formation de l'écrivain et décrire l'évolution de son style à travers les différentes étapes de son œuvre de jeunesse marquée par l'exploration de plusieurs genres littéraires. Pourquoi le jeune écrivain privilégie-t-il deux genres majeurs, le genre autobiographique qui lui permet de concrétiser sa faculté lyrique et le genre théâtral où se révèle sa nature oratoire ? Le style de Flaubert est animé par les deux dimensions lyrique et oratoire, lesquelles dimensions marquent le premier roman de maturité Madame Bovary. Si ce roman marque un tournant dans la carrière de l'auteur c'est d'une part parce qu'il cumule les procédés de l'œuvre de jeunesse et d'autre part constitue le dépouillement du style de cette même œuvre. C'est avec et contre les procédés de l'écriture romantique que Flaubert forgera son style.
\end{abstract}

Mots clés : Evolution, Flaubert, Genre, style, Roman.

\begin{abstract}
The study of Flaubert's early writings is a domain that has not been explored yet. Conducting such a study is so beneficial for grasping the writer's formation and describing the evolution of his style through the different stages of his youthful work, which is marked by the exploration of several literary genres. Why does the young writer privilege two major genres, the autobiographical genre that allows him to concretize his lyric faculty and the theatrical genre in which his oratorical nature is revealed? Flaubert's style is animated by the two lyrical and oratorical dimensions, which characterize maturity of his first novel Madame Bovary. If this novel marks a turning point in the author's career, it is because, on the one hand, it combines the processes of the work of youth, and, on the other hand, it consitutes the emerging style of the same work. It is with and against the processes of romantic writing that Flaubert forges his style.
\end{abstract}

Keywords : Evolution, Flaubert, genre, novel, style.

囷 auteur correspondant : soussou.m@ucd.ac.ma

Pour citer cet article (Style APA) : Soussou, M.Y. (2018). Le « jeune » Flaubert et l'apprentissage des genres littéraires. Francisola: Revue Indonésienne de la langue et la littérature françaises, 3(1), 34-44. doi: 10.17509/francisola.v3i1.11889 


\section{INTRODUCTION}

L'œuvre de jeunesse de Flaubert ne bénéficie pas de l'étude profonde et du grand intérêt qu'on réserve à son œuvre de maturité. Malgré la publication de presque tous les premiers écrits de cet écrivain dans la prestigieuse collection de la pléiade dans le tome 1 de l'édition des CEuvres Complètes chez Gallimard, ces écrits sont toujours négligés et ne sont pas assez explorés particulièrement au niveau stylistique. Mêmes si des revues spécialisées sur Flaubert se trouvent dans le terrain de la recherche scientifique depuis de nombreuses années, pour ne citer que La Revue de Flaubert dirigée par Yvan le Clerc et Flaubert: revue critique et génétique présidée par Pierre Marc de Biasi. Les résultats de l'ensemble de la recherche flaubertienne ne sont pas encore suffisants pour saisir l'acte d'écrire de Flaubert et ce pour une raison fondamentale, c'est que les deux approches, stylistique et génétique, qui permettent de mieux d'interroger le style de cet écrivain sont rarement adoptés par les chercheurs.

Consacrer aujourd'hui un livre, un essai ou un mémoire à Flaubert est une aventure périlleuse car tout travail qui choisit l'œuvre de Flaubert comme objet d'étude doit éviter de menacer ce que Roland Barthes appelle : « l'Irréductible de la littérature ». Flaubert est l'un des premiers écrivains qui ont pensé l'intransitivité de l'écriture, qui ont sapé la conception traditionnelle de la littérature comme représentation d'une fiction. A partir de Flaubert, la littérature devient un travail de réflexion et d'érudition, un travail aussi profond que celui de la science ou de la philosophie. Flaubert est l'un des premiers écrivains qui ont substitué à la question «Pourquoi le monde? » la question « comment écrire? ». Au lieu de changer le monde, Flaubert pense changer la langue. Le problème de l'écriture réside dans le langage, ce problème qui oblige l'écrivain non à écrire avec le langage mais à écrire contre. L'énigme de l'écriture se résume dans la métaphore du chaudron fêlé évoquée dans Madame Bovary, roman qui raconte moins cette banale intrigue d'adultère que l'histoire originale, inédite, et complexe de l'écriture.
Tous les autres romans de Flaubert n'étaient que des tentatives dans lesquelles il a curieusement tenté différentes manières d'écriture, que des épreuves dans lesquelles Flaubert est allé courageusement à la rencontre de cette grave question: Comment écrire?

Toutefois, ce grand programme flaubertien n'a pas été bien reçu et souvent sa portée scripturale n'a pas été bien saisie. L'œuvre de Flaubert a été considérée par ses contemporains comme une stricte application de l'art pour l'art, comme une figuration du réalisme ou comme une préfiguration $\mathrm{du}$ naturalisme, les deux mouvements que Flaubert toutefois abhorre (Faguet, 1899, p.127). A l'exception de Joyce et Proust, écrivains-romanciers de renommée qui au début du XX siècle ont découvert l'originalité de l'écriture de Flaubert et ses usages inédits, les critiques qui ont approché l'œuvre de Flaubert ont laissé de côté ce qui constitue l'apport de cet écrivain dans la modernité romanesque. Il fallait attendre les années 6070 pour que plusieurs facteurs favorisent la reconsidération de l'œuvre de Flaubert : l'essor du structuralisme, l'évolution de la poétique du texte et leur coïncidence avec la commémoration du centenaire de l'Education Sentimentale et avec l'apparition d'une nouvelle génération de critiques, tels que Gérard Genette, Michel Foucauld, Roland Barthes dont les essais étaient marqués par l'intertexte flaubertien, dont la question principale porte moins sur quoi écrit Flaubert que sur la manière avec laquelle il écrit. La redéfinition de l'écriture littéraire va se développer grâce à la fondation de la critique génétique à la fin des années 70. En travaillant sur les manuscrits de Flaubert qui dépassent les 30000 pages, les généticiens sont arrivés à étudier la dynamique complexe de l'écriture de Flaubert, à découvrir la vie d'un Homme voué à l'écriture (de Biasi, 1998, p.87-102). Ces recherches ont abouti à la construction d'une nouvelle poétique, la poétique du manuscrit ou comme le confirme R.D.Genette, «Peut-on aller jusqu'à construire une poétique spécifique du manuscrit qui sera peut être quelque chose comme une poétique de l'écriture, opposée à 
une poétique du texte. » (Genette, 1966, p.2365). Le travail sur le manuscrit permet de dépasser la question de l'origine (à laquelle s'intéresse la philologie ancienne) et de la fin (sacralisée par la poétique du texte). Ce nouvel objet marque ce pont de l'écriture (dont nous ne pourrons jamais délimiter ni le début ni la fin) entre le texte comme une circonstance extérieur et le texte comme un huis-clos. Rien ne peut témoigner des affres de l'écriture mieux que les brouillons. Ce sont les traces matérielles qui racontent l'histoire de l'écriture.

Le thème de la bêtise qui est le plus creusé par ceux qui tendent à approcher l'œuvre de Flaubert sous cet ongle, est souvent étudié dans les romans de maturité alors qu'il convient de retourner aux premiers écrits pour mieux comprendre comment est née chez l'écrivain cette passion pour la sottise humaine et comment le roman deviendra le genre qui lui permet de représenter et d'écrire cette Bêtise et à travers quelles étapes le jeune écrivain passera pour devenir le grand romancier que nous savons. Ce sont les objectifs de notre étude.

Flaubert raille la Bêtise dès son enfance, il va devenir plus tard le romancier de la sottise. Il aurait aimé en être le dramaturge, il aurait pu l'être. Les écrits de jeunesse nous permettent de suivre progressivement la formation de l'artiste, ses débuts littéraires marqués par une conquête systématique et évolutive des genres littéraires et particulièrement son passage de l'écriture théâtrale à l'écriture romanesque.

\section{MÉTHODE}

L'œuvre de jeunesse est considérée comme l'avant-texte des romans de maturité. Ce sont les mêmes thèmes auxquels l'auteur demeure attaché, mais le style est différent et les procédés employés ne sont pas les mêmes. Puisque Flaubert n'emploie pas de brouillons pour rédiger ses premiers écrits, nous ne pouvons pas adopter l'approche génétique pour les étudier (même si plusieurs textes et avant textes de l'œuvre de jeunesse ont été perdus, ces documents n'auraient pu constituer un dossier de genèse complet et suffisant pour les analyser selon cette approche). Nous tenant cependant à les étudier selon l'approche stylistique et en les considérant comme une seule œuvre composée de plusieurs textes, chacun est l'avant-texte ou l'après-texte de l'autre. Nous suivons également deux plans différents, d'une part nous analysons ces textes genre par genre, et d'autre part en respectant la chronologie de leur écriture. La correspondance est un document important pour situer les activités de l'auteur dans le temps exact. Comme dans le cas la surabondante correspondance avec Louise Colet qu'on utilise pour organiser l'évolution de la rédaction de Madame Bovary, pour l'œuvre de jeunesse ce sont les lettres adressées à ses deux amis Ernest Chevalier et Alfred le Poitevin qui nous permettent de nous renseigner sur la formation de la pensée de Flaubert et l'évolution de ses idées philosophiques et esthétiques.

\section{RÉSULTATS ET DISCUSSION}

Dans une de ses premières lettres à Ernest Chevalier, le jeune Flaubert propose à son ami une sorte de collaboration littéraire, «Si tu veux nous associer pour écrire moi, j'écrirait des comédie et toi tu écriras tes rêves, et comme il y a une dame qui vient chez papa et qui nous contes toujours des bêtises je les écrirait. [Les fautes d'orthographes sont celles de Flaubert que Jean Bruneau tend à conserver et à ne pas modifier dans son édition de la Correspondance dans la prestigieuse collection de la Pléiade]» (Bruneau, 1973). La Bêtise est le thème qui l'a séduit précocement et la comédie est le genre qu'il préfère pour mettre en forme les sottises qui l'entourent.

Avant de savoir lire, le très jeune Flaubert a été bercé d'histoires. Le père Mignot, l'oncle d'Ernest Chevalier, lisait à Flaubert à haute voix Don Quichotte, Les lectures de Mignot sont décisives dans la formation de la sensibilité auditive et littéraire de Flaubert (Thibaudet, 1935, p.16). Ses grandes capacités de lecture vont le conduire à attester trop tôt une volonté d'écrire, laquelle volonté est animée par le projet de mettre tout le monde dans un seul livre, c'est ce que Gothot-Mersch (2001) nomme « l'entreprise de totalisation » : 
Beaucoup d'aspects de son œuvre s'expliqueront par ce dessein, et particulièrement par l'entreprise de totalisation que constituent la plupart de ses grands textes. La Tentation de saint Antoine, Salammbô, L'Education sentimentale et Bouvard et Pécuchet tentent, chacun à sa manière, de faire tenir le monde dans un livre. (p.38)

Le théâtre est le lieu adéquat pour représenter ce monde avec toutes ses contradictions. Flaubert possède tous les talents du jeu, de la mise en scène, de la mimique et de la déclamation. Le théâtre du billard que Flaubert construit avec quelques camarades de collège, le personnage du Garçon qu'il fignolait avec ses amis, l'idiot ou l'ours des salons qu'il dansait devant les Goncourt, les œuvres qu'il récitait à haute voix à ses visiteurs, montrent que Flaubert est né pour être un homme du théâtre ; «le fond de ma nature, écrit-il à Louise Collet, le fond de ma nature est quoiqu'on dise, le saltimbanque. J'ai eu dans mon enfance et ma jeunesse un amour effréné des planches » (lettre du 6 ou 7 aout 1846).

Sa passion du théâtre ne se limite pas à l'écriture mais il veut devenir comédien et atteindre la gloire et la célébrité. En 1846, venant de tenir connaissance avec Louise, il rêve de l'éblouir par un succès théâtral, «je ferai jouer une pièce, lui écrit-il, tu serais dans une loge. Tu m'écouterais, tu entendrais m'applaudir » (lettre du 8 ou 9 aout 1846). Ce sont toujours les applaudissements qui enivrent Flaubert dix ans plus tard quand il assiste au succès théâtral de Louis Bouilhet, " je sors de l'odéon, écrit-il à Alfred Baudry, la tête me tourne encore des bruits des applaudissements » (lettre du 6 novembre 1856).

Comment Flaubert va-t-il renoncer au désir de gloire et se consacrer au labeur du style et au souci de l'art.? Au commencement, il y avait chez l'enfant une conscience parfaite de l'existence de différents types d'écrits et cela dès l'âge de neuf ans. Depuis 1831, il expérimentait ardemment divers genres littéraires. La correspondance et les manuscrits largement conservés aujourd'hui témoignent de l'intérêt du jeune Flaubert pour plusieurs formes littéraires: dramatique, romanesque, poétique, didactique, historique, fantastique, autobiographique, etc.

Il annonce à son ami Ernest qu'il a le projet d'un roman, qu'il veut écrire des pièces de théâtre. Les lettres à Ernest dans cette époque du début des années trente révèlent une hésitation entre l'écriture romanesque et l'écriture dramatique, « je t'ai dit, lui-informe-t-il, que je ferai des pièces mais non je ferai des romans que j'ai dans la tête » (lettre du 4 février 1832). C'est le vouloir de tout écrire qui conduit à cette indécision dans le choix de la forme convenable. Tout au long de sa jeunesse, il pratiquera toutes les formes en usage et en mode à l'époque. L'enfant prodigieux tentera même des écrits d'ordre politique, «je t'enveirait [Nous respectons l'orthographe de Flaubert conservée dans l'édition de la pléiade] de mes discours politiques et constitutionnels libéraux » (lettre du 1 janvier 1831), annonce-t-il à Ernest et montrant par l'emploi de tels concepts son initiation précoce à la culture idéologique de son temps. C'est peut-être de l'entourage familial, des journaux lus par ses parents qu'il tire cette connaissance étonnante pour un enfant de cet âge.

Ecrit en juillet 1831, Louis XIII est le premier texte du jeune Flaubert. Son style ne peut être d'un enfant de neuf ans. L'utilisation du passé simple et du présent historique nous révèle qu'un précepteur ou quelqu'un de son entourage l'a aidé pour écrire ce texte. La même chose pour Eloge de Corneille écrit à la première personne et adressé à Corneille lui-même. L'emploi des procédés rhétorique comme dans cette belle phrase éloquente " pour parler de toi, il faudrait des Horaces, des Virgiles, des Homères » (Gothot-Mersch et Sagnes, 2001, p.9), le recours aux interrogations rhétoriques, aux répétitions, aux apostrophes, aux périphrases, aux antithèses, entre autres, révèlent que l'enfant a été initié aux techniques oratoires.

Il avait également une passion pour l'Histoire qui se concrétisera à sa maturité avec la publication de Salammbô, L'Education sentimentale et aussi Hérodias. En 1832, la fondation du «Théâtre de Biallard» avec Ernest avait pour but l'écriture de quelques 
récits historiques sur trois règnes successifs, Henri IV, Louis XIII, Louis XIV.

Le genre allégorique est un autre genre marquant dans l'œuvre de jeunesse. Cette forme d'écriture n'apparait chez Flaubert qu'après ce passage à vide des années 18331834 où il n'écrit pas beaucoup et manque de projets à part un roman historique Isabieu de Bavière dont on n'a trouvé aucune trace. En 1835, il fonde Le Journal des soirées d'études dont on a conservé que le deuxième numéro qui contient Voyage en Enfer, récit allégorique qui traite un sujet très exploité à l'époque, le narrateur accompagné de Satan visitent l'enfer. Le texte d'un lyrisme extrême marqué par l'emploi abusif de «et». Le même sujet sera repris dans La Danse des morts, Smar et La Tentation de Saint Antoine. Flaubert reprend le thème de la mort l'année suivante dans La Femme du monde, une sorte d'autoportrait de la mort où il use de la première personne et d'un ton noir. Le personnage de Satan réapparait l'année suivante (1837) dans Rêves d'enfer où Flaubert exploite cette fois-ci la forme fantastique en introduisant les ingrédients traditionnels du genre (corbeaux, chauves-souris, vieux châteaux, etc.), essayant à travers l'allégorie, à l'aide d'un style ferme, de délivrer un discours sur le monde. Le texte peut être rapproché à Saison en Enfer d'Arthur Rimbaud (1873) d'autant plus que plusieurs ressemblances peuvent nous permettre d'associer les deux «jeunes» expériences flaubertienne et rimbaldienne et la rage du jeune Flaubert à celle du poète maudit.

En ce qui concerne le genre poétique, nous pensons qu'il convient de ne pas étudier les prétendus poèmes de jeunesse de Flaubert signalés périodiquement dans des catalogues, publiés dans des revues ou en volume, et ce pour la raison suivante: ces textes n'ont pas été écrits par Flaubert mais par ces deux amis, Alfred le Poitevin et Luis Bouilhet. Nous devons au premier «La Bayadère », " Les Lotophages », « Comme perce le jour à travers un nuage» entre autres poèmes que René Descharmes reproduit dans son édition d'Une promenade de Bélial ( CEuvres inédites d'Alfred Le Poitevin, PUF,1924) et au second nous reconnaissons d'autres poèmes tels que «Erreur des yeux »,
«A voltaire», «A une femme maigre» attribués par Flaubert lui-même à Bouilhet quand il les cite dans sa préface du recueil poétique de celui-ci intitulé Dernières chansons. D'autres poèmes sont écrits en collaboration avec Bouilhet (La Découverte de la vaccine, Les Litanies de la littérature) ou avec Louise Collet que Flaubert corrige à maintes fois ses poèmes auxquels il ajoute ses propres quatrains.

En 1835, Flaubert essaie cependant l'écriture sentimentale et poétique avec un texte intitulé Une pensée écrit dans un rythme ternaire et consacré à une scène de bal. Il expérimente également l'écriture journalistique tout en adoptant un style bref et en utilisant le «Nous » de majesté comme dans ce texte intitulé « les Echos ». Toujours dans la même année, le jeune Flaubert écrit un drame Frédégonde et Brunehaut dont on a trouvé aucun manuscrit mais il en parle dans sa correspondance (lettre à Ernest chevalier du 18 juin 1835) : «j'ai en tête Frédégonde et Brunehaut, que je m'en occupe (mentalement) depuis environ trois mois, mais surtout depuis 15 jours. Je ne rêve que cela, j'en ai fait une douzaine de lignes, oui ce sera un drame, et autrement fabriqué que les autres ». S'il revient à son genre préféré, « l'adolescent » Flaubert tente cette fois-ci un mélange de formes, une transgression générique et une rénovation stylistique. C'est ce que remarque Gothot-Mersch (2001) :

A treize ans Flaubert a découvert que ce qui fait le grand artiste, c'est la création de formes neuves qui, subvertissant les genres reçus, permettent un renouvellement de notre vision $\mathrm{du}$ monde, ce qui nous vaudra La Tentation de saint Antoine, entre récit, représentation théâtrale et poème ; Salammbô et L'Education sentimentale, romans historiques en infraction avec le genre pour des raisons opposées: l'un trop éloigné dans le temps- ce n'est plus de l'Histoire, c'est de l'archéologie-, l'autre trop proche- ce n'est pas encore l'histoire- sans parler de l'œuvre inclassable que constitue Bouvard et Pécuchet. (p.46)

Le brouillage de la narration et du discours est l'une des caractéristiques de la littérature moderne. Le jeune Flaubert en embrasse les techniques aux années du 
collège (Collège Royal de Rouen, l'actuel Lycée Corneille). Le cahier intitulé «Narrations et Discours» constitue une expérience importante dans le développement de l'apprentissage des formes littéraires chez Flaubert. Ce sont des exercices que le professeur impose à l'élève sur canevas (Gothot-Mersch, 2001, p.47). L'importance de l'enseignement de la rhétorique a contribué dans la formation de l'écrivain Flaubert et a constitué un passage salutaire de l'exercice scolaire aux premières œuvres. Genette (1966) révèle que les écrits de jeunesse de Flaubert sont le produit de la nature de l'enseignement classique de l'époque qui était basé sur l'imitation du style des grands écrivains classique avant qu'il ne cède la place à une autre forme d'enseignement tourné vers l'histoire de la littérature :

Les grands textes de littérature grecque, latine et française n'étaient donc pas des objets d'étude, mais aussi, et de la manière la plus directe, des modèles à imiter. Et l'on sait bien que jusqu'à la fin du siècle (1880) les épreuves littéraires aux compositions, aux examens, au Concours général, furent des poèmes et de discours latins- c'est-à-dire, non des commentaires mais des imitations, des exercices pratiques de littérature. Ce statut ambigu de l'enseignement classique permet donc, chez les plus doués, un passage insensible des derniers exercices scolaires aux premières œuvres; c'est ainsi que les cuvres de jeunesse de Flaubert comprennent cinq «narrations» (cinq contes ou nouvelles historiques et un portrait de Byron) qui sont des devoirs composés en classe de quatrième 1835-1836. (Genette, 1966, p.26)

Tout se mettait d'accord que « Narrations et discours » fut le résultat des exercices stylistiques imposés à l'élève d'abord en cinquième et ensuite en quatrième années du collège. Composé principalement de récits historiques, Le cahier de ces productions nous informe sur la qualité de l'apprentissage rhétorique auquel le collégien était émancipé, sur l'art d'écrire s'appuyant sur les imitations stylistiques au lieu des commentaires historiques.
Si les débuts littéraires de Flaubert dataient des années 1835-1836, les premiers écrits n'en seraient plus une rupture ou une aventure dans le domaine de l'écriture comme c'est le cas aujourd'hui, mais plutôt le prolongement, la continuation et l'aboutissement d'un processus d'apprentissage tellement orienté vers l'art de l'écriture. Jean Bruneau, lui également, insiste sur le rôle de cet enseignement dans la formation esthétique du futur écrivain, cet enseignement dont le contenu est l'exercice de style et dont l'objectif est le développement de l'élocution. L'apprenant est amené à relever les idées d'un texte et les arguments déployés par l'auteur pour les soutenir, ensuite le professeur aide l'élève à orner ces arguments d'une liste de figures de style. «L'élève de rhétorique, écrit Bruneau, apprenait à écrire, au sens fort du verbe, qui est intransitif »(Bruneau, 1962, p.27).

Dans la deuxième moitié du XIX siècle, si la disparition de l'enseignement rhétorique laissera prédominer la fonction descriptive à la place des deux fonctions critique (l'analyse littéraire) et poétique ( la création littéraire) que l'ancienne rhétorique assurait, celles-ci réapparaitront dans des productions littéraires chez Mallarmé, Proust, Valéry, Blanchot, des écrivains qui ont conduit la littérature à réfléchir sur elle-même, et bien évidement récupérer la fonction critique tout en l'associant avec la fonction poétique. Gustave Flaubert était le tremplin vers ce nouveau statut de la littérature se contestant elle-même.

Le jeune Flaubert a été amené à bien écrire, à se préoccuper de la forme au lieu du contenu, à passer de l'écriture de l'histoire à l'histoire de l'écriture et à mettre en œuvre un des grands crédos de son art, à savoir l'intransitivité de l'écriture. Flaubert n'y est pas parvenu du jour au lendemain mais tout au long d'une série d'expériences successives et de recherches insatiables.

A n'observer que les sous-titres des récits historiques pour constater comment Flaubert a consciemment passé du scolaire au littéraire. Loin d'employer des termes vagues comme "rédaction» ou "production", il range des récits comme «Dernière scène de la mort de Margueritte 
de Bourgogne » (Gothot-Mersch et Sagnes, 2001, p.34) parmi les «discours et narrations » série entamée d'août 1835, qualifie $\mathrm{d}^{\prime}$ « opuscules historiques » les deux récits «Mort du duc de Guise » écrit en septembre (Gothot-Mersch et Sagnes, 2001, p.47) et «deux mains sur une couronne » en janvier de l'année suivante (Gothot-Mersch et Sagnes, 2001, p.53), et de «conte historique » un autre récit «Un secret de Philippe Le Prudent, Roi D’Espagne » rédigé en septembre 1836 (Gothot-Mersch et Sagnes, 2001, p.131).

L'emploi d'abord de «narration », puis $\mathrm{d}^{\prime}$ « opuscule » et enfin de « conte» montre un certain passage chez le jeune Flaubert du scolaire au littéraire. Dans ses récits historiques, il abuse du discours direct, les textes sont presque dialogués mais dans certains d'entre eux il développe une forme de récit fait d'une succession de passages narratifs et de passages dialogués notamment dans «Matteo Falcone ». Le style épistolaire apparait dans «Un secret de Philippe Prudent » et annoncé par un soustitre «Les lettres de Charles Quint à Don Juan d'Autriche ». Dans ce dernier récit, le sujet historique est supplanté par le drame psychologique que révèlent les rapports de Philippe II avec son frère ainé et les haines et les violences de la famille royale. « La peste à Florence » ne contient pas de sous-titre qui puisse préciser le genre historique du récit, le titre annonce le thème de la Peste et l'épigraphe suivante (citation d'Alexandre Dumas) dévoile la dimension psychologique et philosophique: "C'est que je te hais d'une haine de frère " (Gothot-Mersch et Sagnes, 2001, p.143).

Parfum à sentir ou les Baladins est un conte philosophique qui techniquement constitue une étape remarquable dans l'évolution stylistique du jeune Flaubert. On y trouve la multiplication des personnages (sept personnages), l'approfondissement de leur description (vestimentaire, physique et gestuelle), le développement des épisodes, du rythme de la phrase, l'élaboration de la frappante chute et particulièrement la variation des points de vue comme dans le texte de la saltimbanque qui nous transporte d'une scène de famille à l'extérieur où un passant entend du dehors sans savoir de quoi il s'agit. Bref, par rapport aux précédents écrits, le progrès est considérable dans la mesure où la composition du récit devient de plus en plus complexe.

Dans La Dernière Heure (1837), un récit autobiographique, après s'être empoisonné, le héros compte le temps qui lui reste pour écrire l'histoire de sa vie, les réminiscences du passé se mêlent aux méditations du présent. Cette autobiographie fictive demeure inachevée et s'arrête sur l'entrée au collège du héros, épisode que l'auteur va relativement développer dans Les Mémoires d'un fou (1838).

Flaubert délaisse le genre autobiographique et essaye « la physiologie » en publiant un texte intitulé «Leçon $\mathrm{d}$ 'histoire naturelle, genre commis » dans une revue locale, Le Colibri, qui a déjà édité Bibliomanie l'année précédente. Le texte écrit sur un ton familier et comique servira dans la genèse de Bouvard et Pécuchet. Il rédige également une étude historique sur «L'influence des Arabes d'Espagne sur la civilisation française au Moyen Age» dans laquelle il glorifie l'apport culturel et artistique de la civilisation arabe en utilisant un style objectif, un ton épique et tout en alternant l'imparfait éternel avec le présent historique :

Adel al-Rahmane I, favorise les lettres et les arts. La puissance qui nous apparait a quelque chose de généreux et de grand. Pendait que les rois des Francs chantaient au lutrin, apprenait à lire, s'étudier à signer son nom, ce calife des Arabes d'Espagne avait a sa cour des savants et des artistes; la collection des canons de l'Eglise se traduisait en arabe; l'arabe et le roman étaient indistinctement parlés. Les savants de l'Europe passaient tous par les académies d'Espagne. Alvaro de Cordoue au IX siècle se plaignait de ce que ces compatriotes parlaient mieux l'arabe que leur langue maternelle. (Gothot-Mersch et Sagnes, 2001, p.1132)

Comparativement aux précédents récits historiques, le style devient de plus en plus coupé, haché. Comme dans ce dernier extrait, L'association des deux auxiliaires, tour flaubertien par excellence, crée une dimension héroïque et mythique aux actions. Le texte devient plus oratoire quand il s'agit 
d'évoquer l'apport de l'architecture arabe, il emploie l'extraction : «quelque chose qui n'a point le génie hébraïque et qui n'ont apporté les Arabes, c'est leur architecture, frêle, audacieuse, sublime, fantastique et maniérée »; le ton devient lyrique quand il passe à vanter le sens poétique, romantique et "féministe"des Arabes, il emploie l'interrogation rhétorique accompagnée d'une subjection mise en relief :

Cette poésie musicale et chantante du Midi, ces notes sonores, cette cadence, ce rythme, cette rime enfin, tout cela nous est venu de l'Orient. Mais l'âme de cette poésie tendre, passionnée et lyrique, le culte rendu à la femme, l'amour enfin sentimentale et langoureux, qui l'a produit? Qui l'a senti le premier? Les Arabes. Nous jugerions fort mal les Musulmans d'après la brutalité jalouse des Turc modernes. Les Arabes laissaient les femmes plus libres que les Ottomans de nos jours. (Gothot-Mersch et Sagnes, 2001, p.1133).

Le sens critique apparait très tôt chez Flaubert, hostile aux idées reçues et aux lieux communs. Pour désigner l'Arabe et le Musulman, L'emploi de la majuscule les préserve dans toute leur altérité et leur différence. Nous retrouverons le même refus des stéréotypes et le même respect de l'Autre dans ses récits de voyage.

Dans la même année (1837), Flaubert esquisse plusieurs scénarios et termine deux récits: Quidquid Volueris et Passion et vertu. Dans le premier, il développe les thèmes romantiques et les modes narratifs si exploités à l'époque comme le récit fantaisiste à la Musset, observation sociale à la Balzac. Le sujet demeure néanmoins original, il s'agit de l'histoire d'un hommesinge, laquelle histoire donne à réfléchir sur l'existence absurde de l'homme, sur sa situation douloureuse et son rejet de la société. Quoique l'auteur établisse une distance envers son personnage en employant la troisième personne, des éléments personnels ponctuent le récit. Djalioh veut tuer sadiquement l'enfant du rival et d'assassiner la femme qui se refuse à lui, c'est le même sentiment de jalousie que l'adolescent Flaubert a ressenti envers Elisa Schlesinger et son mari.
Dans le second récit (Passion et vertu), le sujet est emprunté à un fait divers que Flaubert vient de lire dans un journal local : une femme tue son mari et ses enfants dans le but de s'esseuler avec son amant, mais celui-ci ne voulant plus d'elle, la malheureuse se suicide. Flaubert lui donne le nom de Mazza et à son histoire une dimension psychologique,une femme trompé par le faux amour camouflé dans le discours d'un séducteur. L'histoire de Mazza est une description psychologique de l'amertume de la maitresse délaissée d'autant plus qu'une critique du comportement des hommes envers les femmes. Le texte témoigne de quelque progrès dans la manière avec laquelle le jeune Flaubert multiplie les péripéties du conte, varie la position du narrateur et introduit différents procédés romanesques.

En 1838-1839 et après plusieurs essais embrassant divers genres littéraires, le jeune Flaubert semble atteindre un niveau qu'il lui permet de maitriser deux genres, le genre historique que représente Loys XI et le genre allégorique qui s'épanouit dans Smar. Bien qu'il abandonne ces deux genres pendant de longues années, il reviendra ensuite avec deux œuvres importantes : Salammbô dans le premier genre et La Tentation de saint Antoine dans le second. Par ailleurs, la préface qu'il désire écrire pour Loys XI et le commentaire qu'il veut consacrer sur les défauts de Smar prouvent l'importance de ces deux œuvres dans l'épanouissement de son écriture. Flaubert jugea sévèrement ces deux textes. Selon lui, Loys XI manque de documentation historique et la réflexion philosophique et religieuse dans Smar doit éviter l'écueil de la pensée personnelle.

Le mélange des genres et des registres littéraires deviendra une caractéristique fondamentale de l'œuvre de maturité. Le mélange du grotesque et du tragique le préoccupe dans Loys XI. Il mêle le dramatique et le narratif dans La Danse des morts, texte dans lequel est traité le thème de l'écrivain et de la littérature. Rédigé en 1389, Etude sur Rabelais est un autre essai critique qui comme Eloge de Corneille associe un contenu et une forme dissonants. Sagnes (2001) le considère comme un «discours à 
sujet libre que proposait à cet élève son professeur de rhétorique "le père Magnier"” (p.57).

Quant aux genres personnels tels que le «Cahier intime de 1840-1841 » et le récit de voyage. Le premier regroupe des notes de lectures, des citations, des axiomes, des pensées, des commentaires, des impressions. Les définitions qui le ponctuent nous donnent l'impression de lire un dictionnaire. Le second est composé au terme de ses études secondaires, lorsqu' il voyagea aux Pyrénées et en Corse, à son retour à Croisset il en rédigea sommairement le récit (GothotMersch et Sagnes, 2001, p.645) en s'interrogeant sur la façon dont il convient d'écrire un voyage.

En revanche dans son second voyage en Bretagne avec Maxime Du Camp, il en écrira le récit intitulé : "Par les Champs et par les grèves ", texte qui s'élève au niveau des écrits de voyage de l'époque et par lequel s'ouvre le deuxième volume des CEuvres Complètes de la collection «La Bibliothèque de la pléiade ». Le style et la façon pénible avec lequel est écrit ce récit poussent les éditeurs de cette prestigieuse collection d'intégrer ce récit de voyage dans la phase post-jeunesse, car ce texte constitue un passage décisif vers la maturité, un dépassement de cette allégresse d'écriture qui caractérise toute l'œuvre de jeunesse et une entrée de Flaubert dans « la grande étude dus style ».

Selon Flaubert lui-même, Novembre constitue «la clôture de [sa] jeunesse », c'est la fin d'une étape où la plupart des textes sont marqués par l'inachèvement, le discontinu, la spontanéité, où l'auteur était sous l'influence de la littérature romantique. L'Education sentimentale de 1845 se présente comme une immense avancée dans la conception d'un roman moderne, il y introduit de nouvelles formes, lettres, théâtre de marionnettes, portraits à la Bruyère, etc. nous y trouvons également une réflexion très développée sur la conception de l'artiste qu'il en train de devenir.

Entre 1830 et 1846, Flaubert essayait la plupart des genres littéraires en prose si exploités à l'époque. Cet apprentissage des genres s'est accompagné d'une acquisition progressive des techniques d'écriture et de narration: alternance entre récit et dialogue dans Matteo Falcone, multiplication des scènes et des types de personnages dans $U n$ Parfum à sentir, emploi de l'analepse et d'autres procédés de contrastes pour opposer le présent morne de l'héroïne à sa vie joyeuse d'autrefois.

Quant à la position du narrateur, l'œuvre de jeunesse est dominée par une sorte de narrateur-auteur qui intervient excessivement dans la narration. Ce procédé est fréquent dans la littérature de l'époque, et Flaubert en est influencée d'autant plus que les apostrophes et les invocations du lecteur sont nombreuses dans les premiers écrits avant qu'il ne fasse de l'effacement de l'auteur la règle majeure de son œuvre publiée. A ne comparer que la position du narrateur entre les deux phases pour observer la métamorphose de l'écrivain. Peu à peu, il va rendre plastiques les idées qu'il énonce, au lieu de dire, il va se contenter de montrer. Par exemple, la question du grotesque est exprimée dans la voix du narrateur des Mémoires d'un fou, avec le personnage $\mathrm{du}$ fou du roi dans Loys XI, le personnage de Yuk «ce dieu du grotesque » dans Smar, mais dans La Tentation de saint Antoine, c'est le cochon qui provoquera le grotesque.

A travers ses écrits de jeunesse, Flaubert marque un progrès dans l'emploi du style direct en supprimant de plus en plus les passages dialogués, dans le portrait de ses personnages qu'il évite le plus que possible en le remplaçant par des indices vestimentaires ou gestuels évocateurs et en se débarrassant de la description des personnages secondaires. La construction des épisodes atteint la complexité et le niveau de celles des «comices agricoles» de Madame Bovary.

L'évolution de Flaubert se manifeste dans la longueur des textes que dans la durée de leur écriture : ceux de 1838-1839 sont plus développés que ceux des années 1835-1836, avant que L'Education sentimentale de 1845 n'atteigne le volume des romans de maturité. Les écrits de jeunesse ne contiennent ni brouillons ni plans et se présentent comme des rédactions immédiates de premier jet, le 
jeune Flaubert écrit avec rapidité et se vante d'avoir terminé ses récits dans un temps très court. Les points de suspensions qui $y$ abondent révèlent qu'il ne peut plus suivre le rythme de sa pensée. Les indications chronologiques qu'il transcrit sur ses manuscrits nous renseignement sur la durée de la composition de certaines œuvres, car souvent il mentionne au début de son récit la durée de l'écriture et la date de l'achèvement à la fin. Par exemple, un texte comme Quidquid Volueris est rédigé entre septembre et octobre 1837.

Nous pouvons expliquer cette allégresse d'écriture par l'impatience de la jeunesse et par la faiblesse de la culture théorique et technique. Mais cela est dû aussi à la conception qu'il a lui-même de l'écriture à cette époque. "Je me méfie, écrit-il dans le Cahier intime, des idées et plans préconçus, il faut laisser les idées suivre leur pente, ne pas les forcer » (Gothot-Mersch et Sagnes, 2001, p.740). Avant de proférer l'écriture à programmation scénarique, il a longtemps abusé d'un autre type d'écriture qui est l'écriture à structuration rédactionnelle. de Biasi (2000) établit la différence entre ces deux types d'écriture, (p.27). Avant qu'il adopte l'axiome de Goethe « tout dépend du plan », le jeune Flaubert se lance dans la rédaction sans préparation; à chaque fois qu'il vivait une expérience ou avait une quelconque idée, il s'empresse de l'écrire. Ecrite en novembre et décembre 1837, l'histoire de Mazza racontée dans Passion et vertu est prise d'un fait divers publié en octobre dans Le Journal de Rouen; L'épisode de Caroline dans Les Mémoires d'un fou est rédigé juste après qu'il reçut l'annonce du mariage en janvier 1838 ; quant au passage final de Smar, il le rédigea suite à sa visite d'une maison de prostituée en février 1839.

Les écrits de jeunesse se caractérisent par l'immédiateté et l'inachèvement. Certains d'entre eux ont été abandonnés, par exemple «La Dernière Heure » est inachevé, «deux amours et deux cercueils » est le projet d'un roman qui n'a pas dépassé l'état d'un scénario. Quelques uns manquent de clarté, leur fin est inexpliquée comme celle de Smar ou réorientée comme celle des Mémoires d'un fou, récit interrompu et ensuite repris sans que la deuxième parie ait un rapport avec la première. La dernière partie de Novembre est écrite à la troisième personne alors que le début de cette nouvelle est conçu comme une autobiographie.Ce dernier texte a néanmoins l'avantage de se présenter comme une mise au net dont les brouillons n'ont pas été conservés.

D'autres textes de jeunesse ne manquent pas également d'un travail de structuration et de conception scénarique. Quant à la documentation avant qu'il apparaisse fondamentalement dans la préparation de la première Tentation, ses prémisses s'annoncent déjà dans la préface de Loys XI (Gothot-Mersch et Sagnes, 2001, p.305-306). Flaubert y révèle l'importance de la documentation et sa relation avec l'imagination car l'une excite l'autre comme il l'exprime à Louise Collet dans une lettre du 4 octobre 1846.

\section{CONCLUSION}

Les principes qui constituent plus tard l'éthique de l'art de Flaubert et les particularités qui font de son style un des plus grands écrivains français modernes ont été perçus depuis ses écrits de jeunesse. Les Mémoires d'un fou (1838), Smar (1839), Novembre (1842) et plus encore La premières Education sentimentale (1845) et la première Tentation de saint Antoine (1849) constituent les étapes marquantes de la métamorphose d'un artiste et de l'évolution progressive de sa manière d'écriture. En expérimentant divers genres littéraires, Flaubert découvre peu à peu la prééminence du style et de la forme, l'importance de la documentation et de la recherche. Au lieu de se plaindre selon un topo romantique de l'insuffisance de la langue, de l'inadéquation du réel et $\mathrm{du}$ langage, il apprend à chercher les artifices et les trouvailles stylistiques qui lui permettent d'utiliser ce langage sans être inculpé de la bêtise dans laquelle il est incrusté. Ni le genre théâtral ou poétique ne le satisfont, ni le genre fantastique, allégorique ou d'autres genres chéris par les romantiques ne sauraient contenir ce qui souhaite réaliser. Il découvre que seul le roman peut représenter ce langage en le minant de l'intérieur. Le rêve de Flaubert devient plus grand en aspirant à 
écrire et dans un style parfait ce qu'il appelle la prose poétique ou encore «le livre sur rien ».

\section{RÉFÉRENCES}

Bruneau, J. (1962). Les débuts littéraires de Flaubert. Paris : Armont Colin.

Bruneau, J. (dir.) (1973). Correspondance I Gustave Flaubert (1830-1851). Paris: Gallimard, coll. « Bibliothèque de Pléiade»

de Biasi, P. M. (1988). Flaubert: dynamique de la genèse. Ann. Scuola Norm. Sup. Pisa, 87-102. Repéré à http://www.item.ens.fr/articles-enligne/flaubert-dynamique-de-la-genese/. de Biasi, P.M. (2000). La génétique des textes. Paris : Ed. Nathan.

Faguet, E. (1899). Flaubert. Paris : Librairie Hachette.

Genette, G. (1966). Figures II. Paris : édition du Seuil.

Gothot-Mersch, C., et Sagnes, G. (dir.) (2001). Oeuvres complètes I. Oeuvres de jeunesse. Paris: Gallimard, coll. " Bibliothèque de Pléiade »

Thibaudet, A. (1935). Gustave Flaubert. Paris: Gallimard. 